Computer Science \& Engineering: An International Journal (CSEIJ), Vol.1, No.3, August 2011

\title{
To improve the QoS in MANETs through analysis between reactive and proactive routing protocols
}

\author{
${ }^{1}$ Chandan Kapoor and ${ }^{2}$ Gaurav Sharma \\ ${ }^{1,2}$ Asst. Prof., CSE Department, J M I T RADAUR \\ ${ }^{1}$ chandankapoor.123@gmail.com \\ ${ }^{2}$ gaurav13@gmail. com
}

\begin{abstract}
A Mobile Ad hoc NETwork (MANET), is a self-configuring infra structure less network of mobile devices connected by wireless links. ad hoc is Latin and means "for this purpose". Each device in a MANET is free to move independently in any direction, and will therefore change its links to other devices frequently. Each must forward traffic unrelated to its own use, and therefore be a router. The primary challenge in building a MANET is equipping each device to continuously maintain the information required to properly route traffic. QOS is defined as a set of service requirements to be met by the network while transporting a packet stream from source to destination. Intrinsic to the notion of QOS is an agreement or a guarantee by the network to provide a set of measurable pre-specified service attributes to the user in terms of delay, jitter, available bandwidth, packet loss, and so on. The analysis is mainly between proactive or table-driven protocols like OLSR (Optimized Link State Routing) viz DSDV (Destination Sequenced Distance Vector) \& CGSR (Cluster Head Gateway Switch Routing) and reactive or source initiated routing protocols viz $A O D V$ (Ad hoc on Demand distance Vector) \& DSR (Dynamic Source Routing). The QoS analysis of the above said protocols is simulated on NS2 and results are shown thereby.
\end{abstract}

\section{KEYWORDS}

Mobile Ad hoc Networks (MANETs), Quality of Service (QoS), AODV, DSDV, routing

\section{INTRODUCTION}

Conventional networks typically rely on distance vector or link state algorithms in which periodic advertisements are sent in order to allow all routers to keep routing tables up-to-date. Using these algorithms which ensure that the route to every host is always known, presents several problems. First, periodically updating the network topology increases bandwidth overhead. Secondly, repeatedly awakening hosts to receive and send information quickly exhaust batteries. Then, communication systems often cannot respond quickly enough to dynamic changes in network topology. Two main routing protocols categories are studied in this paper: reactive protocols and proactive protocols. 
Computer Science \& Engineering: An International Journal (CSEIJ), Vol.1, No.3, August 2011

\subsection{REACTIVE ROUTING PROTOCOLS}

Reactive protocols or on-demand routing protocols rather than relying on periodical broadcasts of available routes, discover routes as needed, build and maintain routes. In this case, route to every mobile is not known at any given time. Two different protocols are studied: DSR (Dynamic Source Protocol) and AODV [1] (Ad hoc On-demand Distance vector).

\subsubsection{DSR}

DSR is a simple algorithm based on the concept of source routing: Source nodes determine routes dynamically and only as needed. A source node that wants to send a packet must check its route cache. If there is a valid entry for the destination, the node sends the packet using that route. If no valid route, the source node initiates the route discovery process: it first send a special route request (RREQ) packet to all neighboring nodes and then propagate through the network collecting the address of all nodes visited until it reaches the destination node or intermediate node with a valid route to the destination node. This node initiates the route reply process: it sends a special route reply RREP packet to the source node providing the sequence of all node through which a packet will travel. This algorithm includes also a route maintenance process. Each host sends a route error (RERR) packet if it encounters a broken link. DSR is easily implemented and thus can work with asymmetric links and involves no overhead when there are no changes in the network. Furthermore, it can be improved to support multiple routes from the source to destination. Nevertheless, large bandwidth overhead inherent in source routing. Each route cache collects the addresses of all visited nodes and the RREQ packet can become huge. So, the acceptable network's diameter and its scalability are limited.

\subsubsection{AODV}

A source node that wants to send a message to a destination for which it does not have a route, broadcasts a request RREQ [1] packet. All nodes receiving this packet update their information for the source node and maintain only the next hop's address in a routing table. A RREQ packet contains the source node's address, broadcast ID, current sequence number and the most recent sequence number of the destination node. The response packet RREP is sent by either the destination or a node that has a route to the destination with the sequence number greater than or equal to the sequence number in the RREQ packet. The route is established once the source node receives the RREP. AODV [1] algorithm includes route maintenance facilities. When a link is broken, the related node sends a RERR message to the neighboring nodes using that route. The main advantage of AODV compared to DSR is the reduced bandwidth due to smaller control and data packet [8]. This algorithm has also good scalability because it needs only two addresses: destination and next hop. However, it works with symmetric links and does not allow for multi path routing. So, new routes must be discovered when a link breaks down.

\subsection{PROACTIVE ROUTING PROTOCOLS}

Proactive protocols exploit the periodic exchange of control messages between routers ensuring that the route to every host is always known. This needs high bandwidth overhead. Ad hoc Link state routing algorithm attempts to conserve bandwidth by reducing both the size and the number of control messages. OLSR (Optimized Link State Routing) is the proactive protocol we have 
Computer Science \& Engineering: An International Journal (CSEIJ), Vol.1, No.3, August 2011

studied in this paper.

\subsubsection{OLSR}

OLSR optimizes classic link state routing algorithm in which each node declares all links with neighboring nodes and floods the entire network with routing messages. OLSR protocol uses only multipoint relay nodes (MPR). So, each node maintains a table of MPR selectors and rebroadcast packets received from the originating node (MPR). Periodically, each node broadcasts "hello messages" and selects minimal subset MPR among one-hop neighbors (with symmetric link) to cover all nodes two hops away.

\section{RELATED WORK}

Quality of Service [13] (QoS) refers to a set of mechanisms able to share fairly various resources offered by the network to each application as needed, to provide, if possible, to every application the desired quality [14] (the network's ability to provide a service). The QoS is characterized by a certain number of parameters (throughput, latency, jitter and loss, etc.) and it can be defined as the degree of user satisfaction. QoS model defines architecture that will provide the possible best service. This model must take into consideration all challenges imposed by Ad-hoc networks, like network topology change due to the mobility of its nodes, constraints of reliability [9] and energy consumption [10], so it describes a set of services that allow users to select a number of safeguards (guarantees) that govern such properties as time, reliability, etc. New requirements (needs) for multimedia and real-time applications require few delay [9] and very high data rates which require (oblige) the use of new routing protocols supporting QoS. The AODV protocol (Ad-hoc on demand Distance Vector) is a reactive routing protocol based on the distance vector Principle, combining unicast and multicast [11] routing. In AODV, the path between two nodes is calculated when needed (if necessary), i.e. when a source node wants to send data packets to a destination, it finds a path (Discovery Phase), uses it during the transfer phase, and it must maintain this path during its utilization (Maintenance Phase). The finding and maintaining process of a path is based on the exchange of a set of control packets: RREQ (Route REQueset), RREP [1] (Route Reply), RERR (Route Error), RRepAck (Route Reply Acknowledgment) and Hello messages (Hello). RREQ is initiated by the source node to find a path in multicast mode [15]. RREP is used by an intermediate or destination node to respond to a request of path finding in unicast mode. Hello messages are used to maintain the consistency of a previously established path. Routing table is associated for each node in AODV protocol with containing: the destination address, the list of active neighbors, the number of hops (hop) to reach the destination, time of expiration after which the entry is invalidated, and so on. To avoid the formation of infinite loop, AODV uses the principle of sequence numbers, limiting the unnecessary transmission of control packets (problem of the overhead); these numbers allow the use of fresh routes following the mobility of nodes, as they ensure the coherence and consistency of routing information.

The Dynamic Source Routing (DSR) is one of the purest examples of an on-demand routing protocol that is based on the concept of source routing. It is designed specially for use in multihop ad hoc networks of mobile nodes. It allows the network to be completely self organizing and self-configuring and does not need any existing network infrastructure or administration. DSR uses no periodic routing messages like AODV [3], thereby reduces network bandwidth overhead, conserves battery power and avoids large routing updates. Instead DSR needs support from the MAC layer to identify link failure. DSR is composed of the two mechanisms of Route Discovery 
Computer Science \& Engineering: An International Journal (CSEIJ), Vol.1, No.3, August 2011

and Route Maintenance, which work together to allow nodes to discover and maintain source routes to arbitrary destinations in the network. DSR has a unique advantage by virtue of source routing. As the route is part of the packet itself, routing loops, either short - lived or long - lived, cannot be formed as they can be immediately detected and eliminated. This property opens up the protocol to a variety of useful optimizations. Neither AODV nor DSR guarantees shortest path. If the destination alone can respond to route requests and the source node is always the initiator of the route request, the initial route may the shortest.

OLSR makes use of "Hello" messages to find its one hop neighbors and its two hop neighbors through their responses. The sender can then select its multipoint relays (MPR) based on the one hop node that offers the best routes to the two hop nodes. Each node has also an MPR selector set, which enumerates nodes that have selected it as an MPR node. Reactive routing protocols do not maintain routes, but build them on demand. As link-state protocols require database synchronization, such protocols typically use the distance vector approach, as in AODV and DSDV, or more ad-hoc approaches that do not necessarily build optimal paths, such as Dynamic Source Routing. OLSR uses Topology Control (TC) messages along with MPR forwarding to disseminate neighbor information throughout the network. Host and Network Association (HNA) messages are used by OLSR to disseminate network route advertisements in the same way TC messages advertise host routes as shown in Figure 1 and 2.

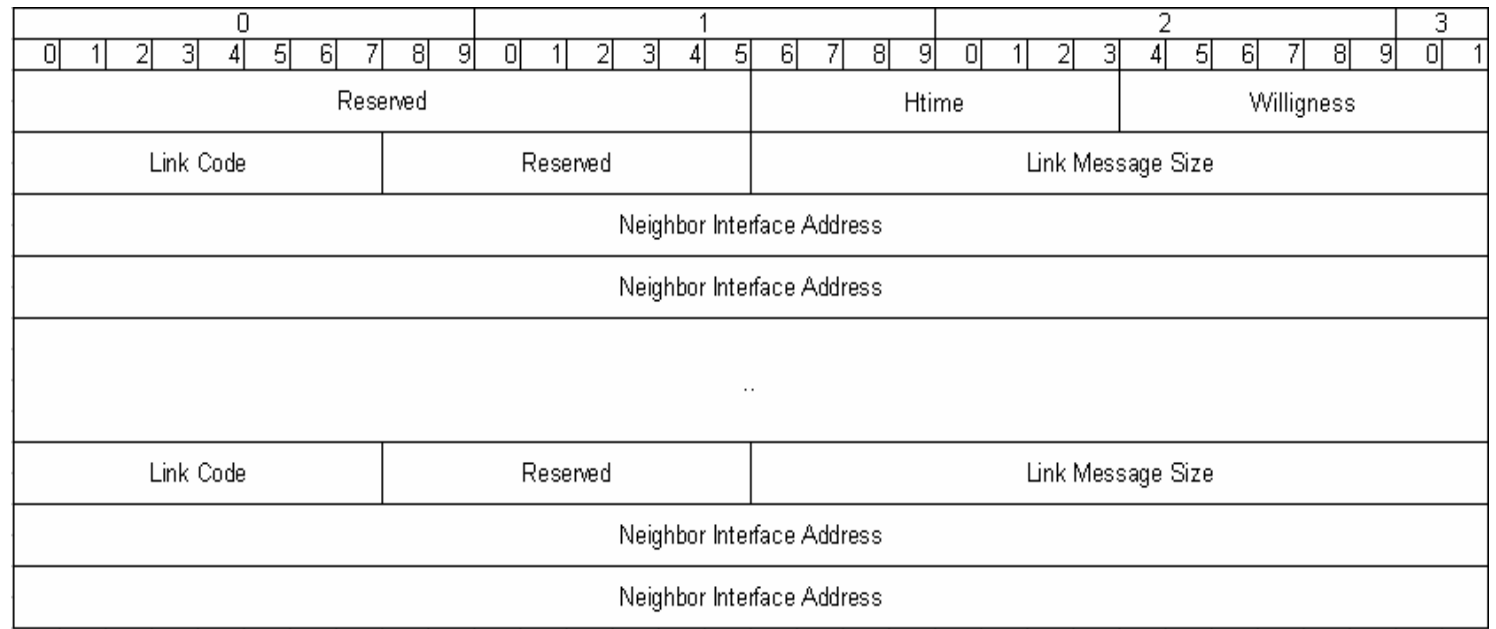

Fig 1. Hello message in OLSR

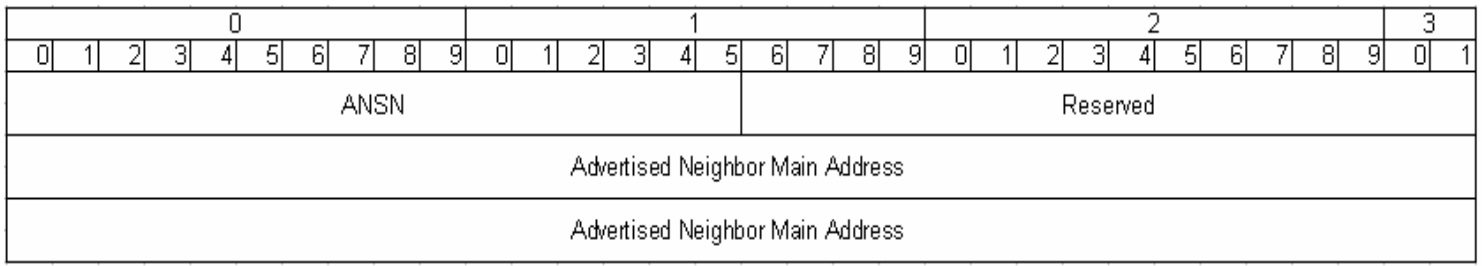

Fig 2. Topology Control (TC) messages in OLSR

OLSRv2 is currently being developed within the IETF. It maintains many of the key features of the original including MPR selection and dissemination. Key differences are the flexibility and modular design using shared components: packet format packetbb, and neighborhood discovery protocol NHDP. These components are being designed to be common among next generation 
Computer Science \& Engineering: An International Journal (CSEIJ), Vol.1, No.3, August 2011

IETF MANET [12] protocols. Differences in the handling of multiple address and interface enabled nodes is also present between OLSR and OLSRv2

\section{OUR PROPOSAL}

The traditional QOS approaches are loosely based on the virtual circuit model that requires explicit connection management and the establishment of hard-state in the network prior to communication. The virtual circuit model also assumes the route and the reservation between source-destination pairs remain fixed for the duration of a session. However, the virtual circuit lacks the intrinsic flexibility needed to adapt to the dynamics found in mobile ad hoc networks where the path and reservation need to dynamically respond to topology and resource changes in a timely manner. Thus, this proposed approach gives a comparative study of the proactive and reactive routing protocols and works for the most challenging aspects of QoS [6] support in mobile ad hoc networks as in the maintenance of service level. In this study, we focus on two mobile node mobility models to simulate their movement. First of them is the Proba Walk mobility model. This model uses a probability matrix to determine the node new position. Three states are used in order to determine the next position. state 0 represents the current value $\mathrm{x}$ or $\mathrm{y}$, state 1 represents the previous $\mathrm{x}$ or $\mathrm{y}$ position and state 2 represents the mobile node next position if the mobile moves in the same direction. Each node moves using an average speed and four possible directions (i.e., north, south, east, or west). The Proba Walk model gives more realistic behaviors compared to random movements. The second mobility model is the Modi_ed Random Direction model which was created to avoid the clustering mobile node near the center of the simulation area. This behavior occurs in the random waypoint mobility direction. In the Modified Random Direction mobility model, each mobile node chooses a random direction and travels over a random distance at a random speed to it. After being arrived, it pauses for a specific time and then chooses a new direction. If it reaches the edge of the simulation area, it bounces. To ensure that sufficient bandwidth is available on the end-to-end path, the RREQ must traverse the complete route to the destination. For this reason, no intermediate node is allowed to reply to the RREQ; only the destination may respond. Also, traffic [4] from different sources to the same destination may need to be forwarded along different paths based on session requirements and resource availability. For this reason, Q-AODV discovers and maintains routes on a persource- destination-pair basis rather than a per-destination basis as in AODV. In Q-AODV, unlike AODV, it is beneficial for the destination to reply to multiple RREQ instances, since the first RREQ instance to reach the destination need not have traveled along the most resourceful path; another path with higher bandwidth availability may be present. The source can then select the best path from among the RREPs received. After selecting the best path, the source informs the destination of its decision by sending a message along the path, so that the destination can use the same path for reverse communication. To provide quality of service (QoS) through minimizing interference is particularly challenging for mobile ad hoc networks [2](MANETs) due to frequent movement and formation of dynamic connections. Therefore, interference from mobility must be overcome to obtain practical QoS in ad hoc networks. In particular, for far away nodes, nodes in ad hoc networks directly communicate with other nodes in a multi-hop fashion: each node operates as a router (or a forwarder) if it is not the destination node. However, connection loss may occur due to interference, which can cause the transmission routes to become out-of-date and inaccurate. This can then result in a serious damage in terms of global network throughput and delay [9]. 
Computer Science \& Engineering: An International Journal (CSEIJ), Vol.1, No.3, August 2011

Different protocols are then evaluated based on measure such as the packet drop rate, the overhead introduced by the routing protocol, end-to-end packet delays, network throughput etc., thus it is quite necessary to study the performance of QoS [5] parameters for both types of routing protocols vis-à-vis reactive routing and proactive routing protocols . The main focus of our study is the analysis of AODV, DSR and OLSR routing protocols so that performance evaluation can be done effectively.

The comparison between the three routing protocols is shown below in Table 1 .

\begin{tabular}{|c|c|c|c|}
\hline Parameters & AODV & DSR & OLSR \\
\hline $\begin{array}{c}\text { Source } \\
\text { Routing }\end{array}$ & No & Yes & No \\
\hline Topology & Full & Full & Reduced \\
\hline Broadcast & Full & Full & Local \\
\hline $\begin{array}{c}\text { Update } \\
\text { information }\end{array}$ & $\begin{array}{c}\text { Route } \\
\text { error }\end{array}$ & $\begin{array}{c}\text { Route } \\
\text { error }\end{array}$ & $\begin{array}{c}\text { Node's } \\
\text { height }\end{array}$ \\
\hline $\begin{array}{c}\text { Update } \\
\text { destination }\end{array}$ & Source & Source & Neighbors \\
\hline Method & Unicast & Unicast & Broadcast \\
\hline $\begin{array}{c}\text { Storage } \\
\text { Complexity }\end{array}$ & $\mathrm{O}(\mathrm{E})$ & $\mathrm{O}(\mathrm{E})$ & $\mathrm{O}\left(\mathrm{Dd}^{*} \mathrm{~A}\right)$ \\
\hline
\end{tabular}

Table 1. Comparison between AODV, DSR and OLSR
Abbreviations:
Dd - Number of maximum desired destinations
E-Communication pairs
A - Average number of adjacent nodes

\section{SIMULATION RESULTS AND OBSERVATIONS}

In this paper we focus on Constant Bit Rate (CBR) sources (i.e voice sources) and ftp sources (i.e. file transfer). The packet size is limited to 512 bytes. The source-destination pairs are chosen randomly over the network. The source-destination numbers are fixed (called connection number). We make the offered load vary by using scenarios with $10,20,30,40,50$ and 60 connections. Each source-destination pair begins packet sending at a chosen time and keeps sending between 40 and 80s for CBR sources and between 5 and 20 for ftp sources. Some parameters are used in order to make performance evaluation of routing protocols in ad hoc mobile network environment. They are resumed in the following table as shown below in Table 2 . 
Computer Science \& Engineering: An International Journal (CSEIJ), Vol.1, No.3, August 2011

\begin{tabular}{||c||c|c||}
\hline \multicolumn{1}{|c||}{ parameters } & CBR traffic & ftp traffic \\
\hline \hline node number & $125,150,175,200,225,250,275$ & $125,150,175,200,225,250,275$ \\
\hline field size $(\mathrm{m})$ & 1100 & 1100 \\
\hline simulation duration $(\mathrm{s})$ & 180 & 180 \\
\hline number of connection & $10,20,30,40,50,60$ & $10,20,30,40,50,60$ \\
\hline Mac Layer & IEEE 802.11 & IEEE 802.11 \\
\hline communication range $(\mathrm{m})$ & 100 & 100 \\
\hline min transfer duration $(\mathrm{s})$ & 40 & 5 \\
\hline max transfer duration $(\mathrm{s})$ & 80 & 20 \\
\hline input rate per connexion $(\mathrm{Kb} / \mathrm{s})$ & 8 & 64 \\
\hline
\end{tabular}

Table 2. The mean CBR and ftp parameters

\subsection{PERFORMANCE INDICES}

We have measured the following performance metrics to evaluate the effectiveness and efficiency of the above mentioned protocols.

4.1.1 Packet delivery fraction: This is the fraction of data packets sent by the server that arereceived by the client. The higher the packet delivery fraction, the more effective the protocol in reducing network congestion and the better the user experience in viewing/hearing the media stream.

4.1.2 Mean end-to-end packet delay: This is the average end-to-end delay of packets that are received by the client. As this value decreases, the congestion in the network is reduced, and packets are more likely to be received in time for playout.

4.1.3 Routing Load: It gives the number of routing packets over the number of received data packets. Each routing packet sent or forwarded by a mobile is counted.

4.1.4 Jitter: It gives the transmission delay variation. Packets from the source will reach the destination with different delays. A packet's delay varies with its position in the queues of the routers along the path between source and destination and this position can vary unpredictably

\subsection{RESULTS}

Several simulations are performed using NS2 network simulator and using parameters shown in table 2. NS2 generates a big trace files analyzed using a statistical tools developed in java. The performance study concerns three routing protocols DSR, AODV [7] and OLSR described in the second section and both CBR and ftp traffic sources. We propose here to study the impact of traffic load by varying the number of connections (pair of source and destination). The following figures show performance evaluation of AODV, DSR and OLSR protocols related to $10,20,30,40,50$ and 60 connections and 200 mobile nodes. In1 authors show that the mobility models may drastically affect protocol performances. So, using the Proba Walk mobility model was conducted by study walker scenarios aim in this part of a paper. We first observe in figure 1 
Computer Science \& Engineering: An International Journal (CSEIJ), Vol.1, No.3, August 2011

that AODV outperform DSR and OLSR for packet delivery ratio (3 to 5\% compared to OLSR) and also routing load criteria as shown in figure 7 (between 4 and 12 times lower). As shown in figures 3 and 5, OLSR performs better and gives little variation when the traffic load increases. Until 30 connections, all studied routing protocols perform identically but when the traffic load increases, OLSR performs better for delay and jitter [9]. However, routing load is higher (see figures 7 and 8) because it periodically sends routing packets in order to maintain the routing table up-to-date. We also observe packet delivery ratio higher (about 98\%) compared to CBR sources because ftp uses TCP protocol which insures packets retransmission when dropped.

In order to study the impact of the node mobility against ad hoc routing protocols, we have pitched on the Modified Random Direction mobility. The mean feature of the model is traveling on all simulation area which avoiding nodes centralization in a specific zone. Some parameters related to this model are resumed in table 3 .

\begin{tabular}{|c|l|}
\hline parameters & values \\
\hline \hline step interval $(\mathrm{m})$ & {$[50,100]$} \\
\hline speed interval $(\mathrm{m} / \mathrm{s})$ & {$[2,15]$} \\
\hline pause_time $(\mathrm{s})$ & $0,5,10,15,20,25$ \\
\hline
\end{tabular}

Table 3. The Modified Random Direction parameters

The performance analysis graphs are as follows:

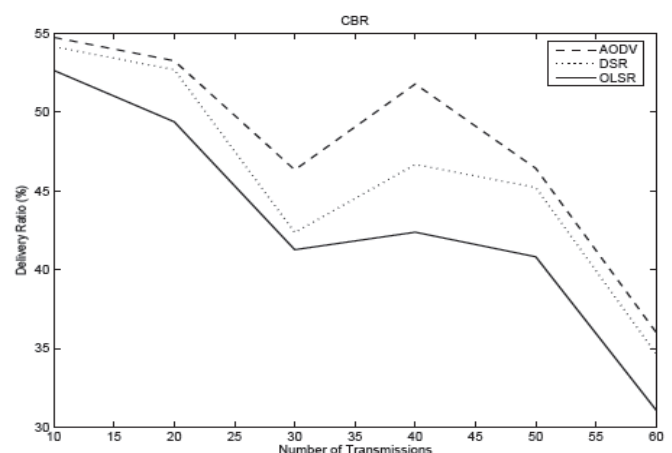

Fig.1 N = 200, Proba Walk mobility model

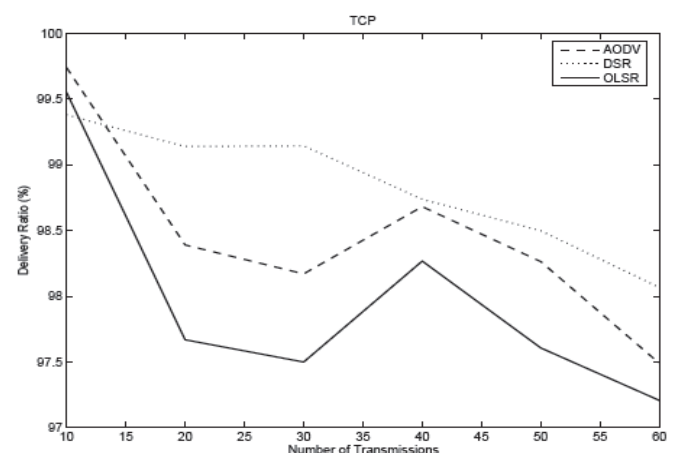

Fig. $2 \mathrm{~N}=200$, Proba Walk mobility model 
Computer Science \& Engineering: An International Journal (CSEIJ), Vol.1, No.3, August 2011
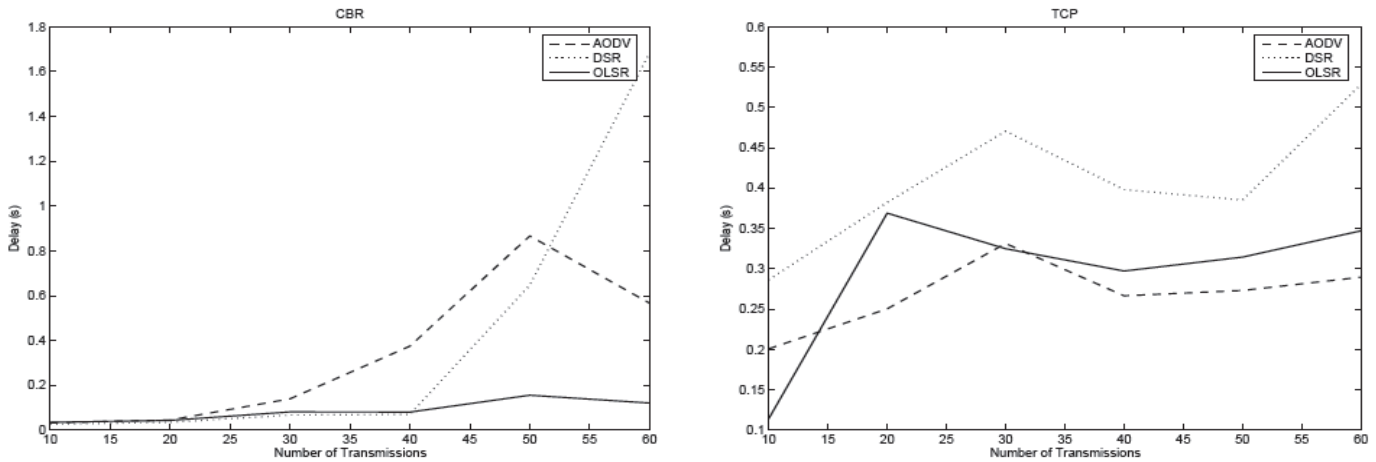

Fig.3 N = 200, Proba Walk mobility model

Fig.4 = 200, Proba Walk mobility mode
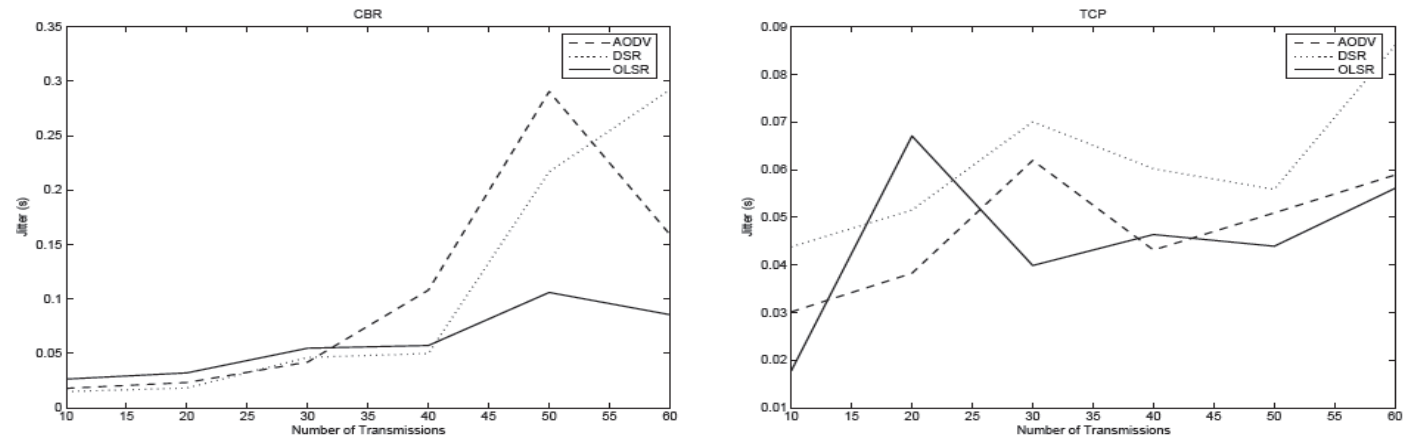

Fig.5 N = 200, Proba Walk mobility model

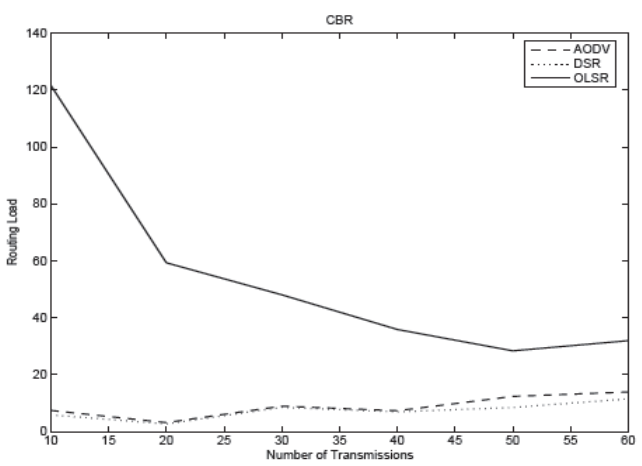

Fig.6 N = 200, Proba Walk mobility model

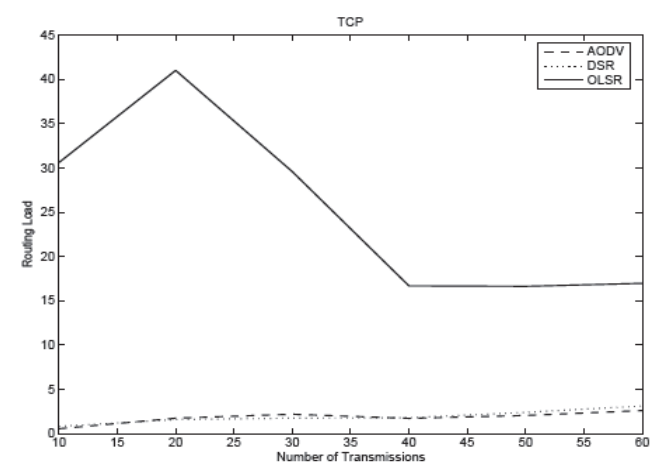

Fig.7 N = 200, Proba Walk mobility model

Fig. $8 \mathrm{~N}=200$, Proba Walk mobility model 
Computer Science \& Engineering: An International Journal (CSEIJ), Vol.1, No.3, August 2011

The figure 9 show the small increases of packet delivery ratio when the mobility decreases (pause time increases between 10 and 20). However, we show in figures 15 and 16 an important decreases of routing load (about $50 \%$ for OLSR). The best performance is given by the AODV protocol because it need lowest routing load for routes discovery. The most important criteria for real-time services is delay and jitter. So, OLSR gives better performance results compared to both DSR and AODV (figures 13 and 15).

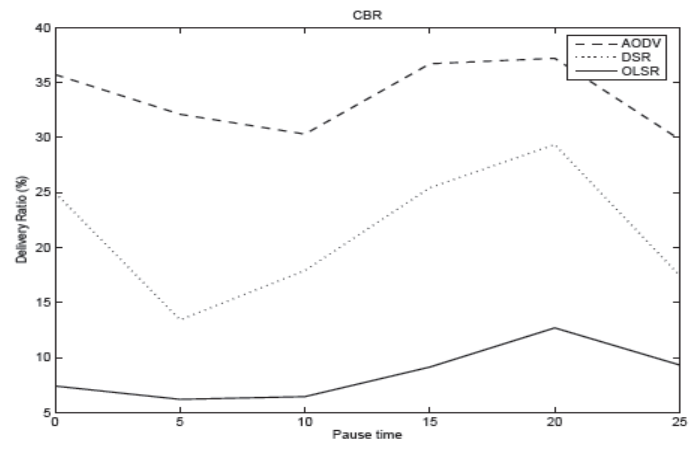

Fig,9. 40 connections, MRD mobility model

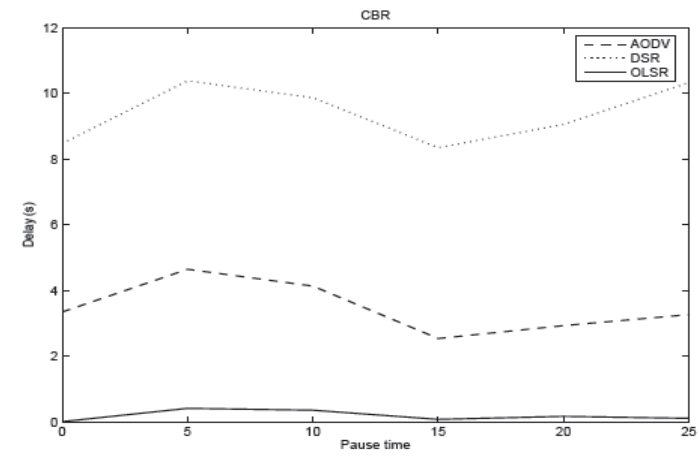

Fig,11. 40 connections, MRD mobility model

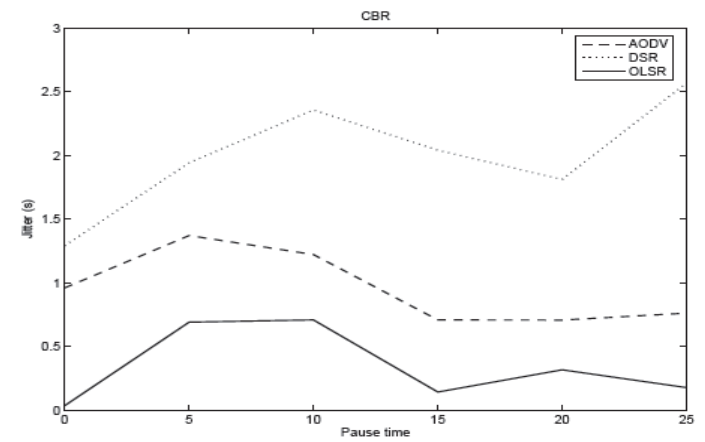

Fig,13. 40 connections, MRD mobility model

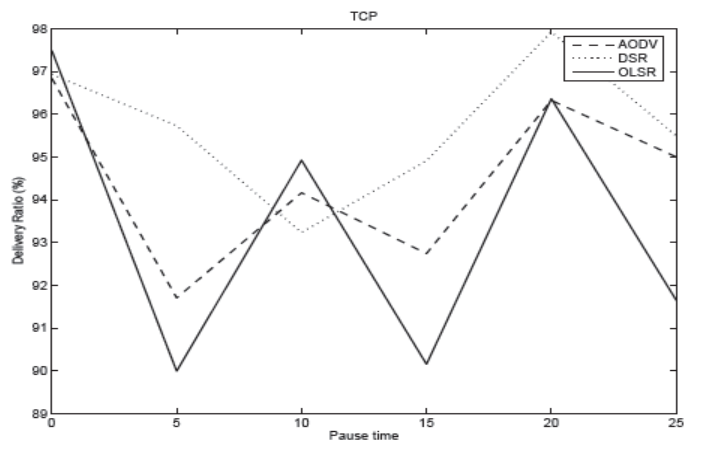

Fig.10 40 connections, MRD mobility model

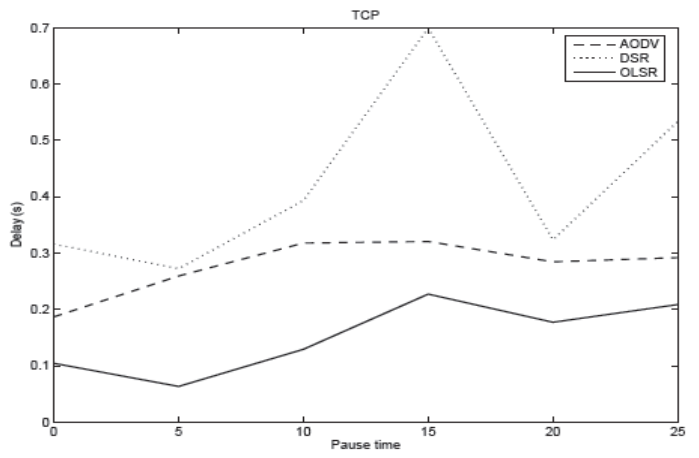

Fig.12 40 connections, MRD mobility model

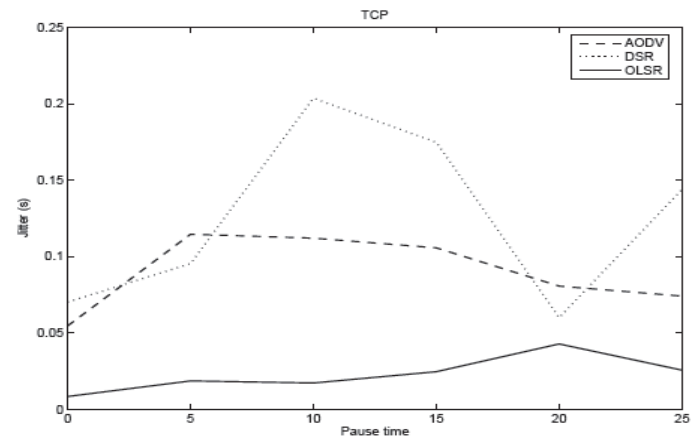

Fig.14 40 connections, MRD mobility mode 


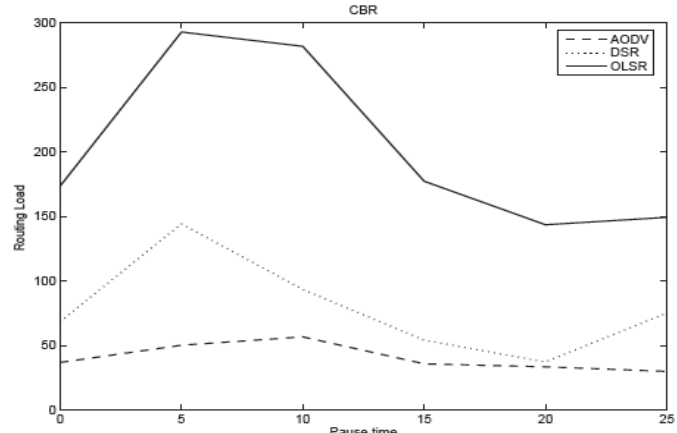

Fig, 15.40 connections, MRD mobility model

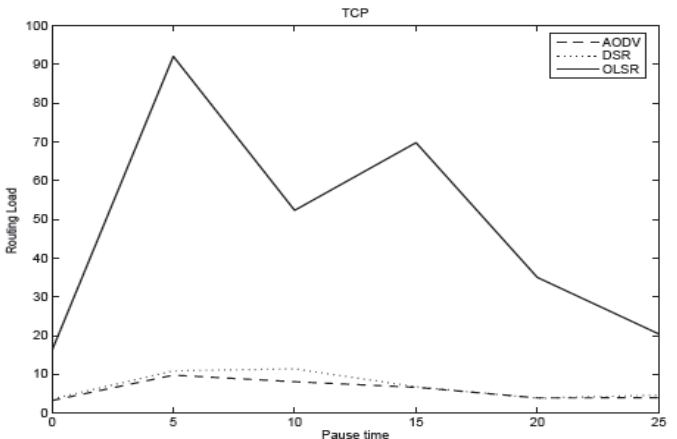

Fig.16. 40 connections, MRD mobility model

In this study, we used the Modified Random Direction model. The first observation concerns OLSR which is the best running protocol for voice services (i.e CBR sources). The packet delivery ratio, delay and jitter are the lowest but the routing ratio is highest (figures 19,21, 23 and 25. It also seems that the network density (i.e number of nodes) in OLSR has no impact on the packet delivery ratio and mean delay criteria while routing ratio increases with the number of nodes. In reactive routing protocols (i.e DSR and AODV), the packet delivery ratio increases when the density of network increases. In addition, proactive routing algorithm (i.e OLSR) produces lower delay and jitter (mean QoS for voice services). This is due to much greater number of nodes which is concerned with the routing table's update. So, number of sending routing packets logically increases.

\section{CONCLUSION}

In this paper we have evaluated the performance of reactive (i.e. DSR and AODV) and proactive (i.e. OLSR) routing protocols in 802.11 ad hoc network environment. We have noticed the proactive protocol (OLSR) offers better performances for CBR sources (e.g. voice services) given that it guaranties lowest delay and jitter. However it consumes much more bandwidth. Periodically, OLSR protocol sends routing packets to discover and to maintain routes to all destinations. That's why the number of delivered packets decreases when the traffic load (number of connections) increases. For 10 connections, the packet delivery ratio is about $53 \%$. The reactive routing protocols are more adapted for data services (file transfer). They guarantee a packet delivery ratio of $80 \%$ for 60 connections ( $480 \mathrm{kbit} / \mathrm{s})$. There is no clear winner among DSR and AODV since routing load, delay and jitter are quite identical. We have also pointed out the influence of the node mobility model on the ad hoc routing protocols. In Proba Walk mobility, direction range is indeed more limited than in Modified Random Direction model.

\section{REFERENCE}

[1] Shakeel Ahmed and A. K. Ramani, "Alternate Route for Improving Quality of Service in Mobile Ad hoc Networks", IJCSNS International Journal of Computer Science and Network Security, VOL.11 No.2, February 2011.

[2] Yu Ping and Wang Ying, "A Revised AODV Protocol with QoS for Mobile Ad hoc Network", 978-1 4244-4520-2/09/@2009 IEEE 
Computer Science \& Engineering: An International Journal (CSEIJ), Vol.1, No.3, August 2011

[3] Vijayalaskhmi M., Avinash Patel and Linganagouda Kulkarni, "QoS Parameter Analysis on AODV and DSDV Protocols in a Wireless Network", International Journal of Communication Network \& Security, Volume-1, Issue-1, 2011

[4] Kyungjun Kim, "A distributed channel assignment control for QoS support in mobile ad hoc networks", Journal of Parallel and Distributed Computing 71 (2011) 335-342.

[5] J.Premalatha and P.Balasubramanie (2010), "Enhancing Quality of Service in MANETS by Effective Routing", 978-1-4244-5137-1/10/2010 IEEE

[6] C.Zhu and S. CorsonC, "QoS Routing for Mobile Ad Hoc Networks”, IEEE Infocom, 2002.

[7] C.Perkins and E. Royer, “Ad-hoc On-Demand Distance Vector Routing”, IEEE WMCSA, 1999

[8] Adib M.Monzer Habbal and Suhaidi Hassan, "Loss Detection and Recovery Techniques for TCP in Mobile Ad Hoc Network", SMIEEE in 2010 Second International Conference on Network Applications, Protocols and Services.

[9] K. Kang and H.-J. Suh, "Adaptive buffer control to minimize delay and guarantee service reliability", The Institution of Engineering and Technology (IET) Communications, 2011, Vol. 5, Issue 1, pp. $110-118$

[10] N.Sumathi and Dr. Antony Selvadoss Thanamani, "Evaluation of Energy Efficient Reactive Routing Protocols in QoS Enabled Routing for MANETS", IJCA (0975 - 8887) Volume 14- No.2, January 2011

[11] Thomas J. Sapienza, "Optimizing Quality of Service of Wireless Mobile Ad-hoc Networks using Evolutionary Computation”, CSIIRW '08, May 12-14, Oak Ridge, Tennessee, USA.

[12] K. Dridi, K. Djouani and B. Daachi, "Three Scheduling-levels algorithm for IEEE 802.11 QoS efficiency improvement", Proceedings of MoMM2008, Linz, Austria.

[13] Roch A. Guerin and Ariel Orda, "QoS Routing in Networks with Inaccurate Information: Theory and Algorithms", IEEE/ACM TRANSACTIONS ON NETWORKING, VOL. 7, NO. 3, JUNE 1999

[14] Larry C. Llewellyn, Kenneth M. Hopkinson, Member, IEEE, and Scott R. Graham, Member, IEEE, "Distributed Fault-Tolerant Quality of Wireless Networks", IEEE TRANSACTIONS ON MOBILE COMPUTING, VOL. 10, NO. 2, FEBRUARY 2011.

[15] Mohammad M. Qabajeh, Aisha-Hassan A. Hashim, Othman Khalifa and Liana K. Qabajeh, "Position- based QoS Multicast Routing Protocol for Mobile Ad Hoc Networks", International Conference on Computer and Communication Engineering (ICCCE 2010), 11-13 May 2010, Kuala Lumpur, Malaysia 